\title{
A competência relacional de enfermeiros em unidades de centros cirúrgicos
}

\author{
Relational competence of nurses in surgical center units \\ Competencia relacional de enfermeras em unidades de centros quirúrgicos
}

\author{
Danilo José dos Santos'; Silvia Helena Henriques"'; Laura Andrian Leal'I'; \\ Mirelle Inácio Soares'v; Lucieli Dias Pedreschi Chavesv'; Beatriz Regina da Silva ${ }^{V I}$
}

\begin{abstract}
RESUMO
Objetivo: identificar e descrever como é desenvolvida a competência relacional no cotidiano do trabalho de enfermeiros cirúrgicos e identificar em que momento/situações o enfermeiro utiliza essa competência. Método: estudo exploratório, qualitativo. O cenário foram unidades cirúrgicas pertencentes a cinco instituições hospitalares privadas de um município no Estado de Minas Gerais e a amostra composta por 43 enfermeiros atuantes destas unidades. Foi realizada a técnica de grupo focal em 2018 e os dados foram interpretados pela análise temática indutiva. Resultados: evidenciou-se que a competência relacional se dá por meio do desenvolvimento de capacidades a serem adquiridas pelos enfermeiros, tais como o gerenciamento de conflitos, comunicação assertiva, gestão de pessoas por meio do dimensionamento de pessoal e inteligência emocional. Considerações finais: $\mathrm{O}$ ambiente cirúrgico demanda atividades que potencializam o desgaste físico e emocional do enfermeiro com demais membros da equipe de saúde, o que torna imprescindível o aprimoramento de capacidades/aptidões associadas a competência relacional.
\end{abstract}

Descritores: Enfermagem Perioperatória; Relações Interpessoais; Comunicação; Competência Profissional; Inteligência emocional.

\section{ABSTRACT}

Objective: to identify and describe how relational competence is developed in surgical nurses' day-to-day work and identify when and in what situations nurses use this competence. Method: qualitative, exploratory study. The scenario were surgical units at five private hospitals in a municipality in Minas Gerais, and the sample comprised 43 nurses working in these units. The focal group technique was applied in 2018 and the data were interpreted by inductive thematic analysis. Results: relational competence was found to be developed by the nurses' acquiring and developing capacities/skills, such as conflict management, assertive communication, people management by appropriate personnel scaling, and emotional intelligence. Final considerations: the surgical environment demands activities that heighten nurses' physical and emotional exhaustion, and its effects on other members of the health team, making it essential to improve capacities/skills in relational competence.

Descriptors: Perioperative Nursing; Interpersonal Relations; Communication; Professional Competence; Communication; Emotional Intelligence.

\section{RESUMEN}

Objetivo: identificar y describir cómo se desarrolla la competencia relacional en el trabajo diario del enfermero quirúrgico e identificar cuándo y en qué situaciones el enfermero utiliza esta competencia. Método: estudio exploratorio cualitativo. El escenario fueron unidades quirúrgicas de cinco hospitales privados de un municipio de Minas Gerais, y la muestra estuvo conformada por 43 enfermeras que laboran en estas unidades. La técnica del grupo focal se aplicó en 2018 y los datos se interpretaron mediante análisis temático inductivo. Resultados: se encontró que la competencia relacional es desarrollada por las enfermeras adquiriendo y desarrollando capacidades / habilidades, tales como manejo de conflictos, comunicación asertiva, manejo de personas por escalamiento apropiado del personal e inteligencia emocional. Consideraciones finales: el ambiente quirúrgico demanda actividades que aumenten el agotamiento físico y emocional del enfermero, y sus efectos sobre otros miembros del equipo de salud, por lo que es fundamental mejorar las capacidades / habilidades en la competencia relacional. Descriptores: Enfermería Perioperatoria; Relaciones Interpersonales; Comunicación; Competencia Profesional; Relaciones Interpersonales; Comunicación; Inteligencia emocional.

\section{INTRODUÇÃO}

Em uma instituição hospitalar, a Unidade de Centro Cirúrgico (UCC) constitui unidade complexa decorrente da tecnologia disponível, da variação intrínseca nos seus processos de trabalho e da situação de vulnerabilidade dos pacientes ${ }^{1}$. A equipe de enfermagem neste setor representada por um contingente expressivo de profissionais é gerenciada por enfermeiros que dependendo da forma como organiza o seu trabalho pode interferir na qualidade do cuidado prestado incidindo, assim, de maneira relevante na organização.

'Graduando de enfermagem. Universidade de São Paulo. Ribeirão Preto, São Paulo, Brasil. E-mail: danilojose@usp.br. ORCID: https://orcid.org/0000-0003-1056-2595 "Enfermeira. Professora Associada. Universidade de São Paulo. Ribeirão Preto, São Paulo, Brasil. E-mail: shcamelo@eerp.usp.br. ORCID: https://orcid.org/0000-0003-2089-3304 I'Enfermeira. Doutoranda. Universidade de São Paulo. Ribeirão Preto, São Paulo, Brasil. E-mail: laura.andrian.leal@usp.br. ORCID: https://orcid.org/0000-0002-8563-8980 IVEnfermeira. Docente Titular. Centro Universitário de Lavras. Lavras, Minas Gerais, Brasil. E-mail: mirelleenfermagem@gmail.com. ORCID: https://orcid.org/0000-0002-5298-8634 VEnfermeira. Professora Associada. Universidade de São Paulo. Ribeirão Preto, São Paulo, Brasil. E-mail: dpchaves@eerp.usp.br. ORCID: https://orcid.org/0000-0002-8730-2815 VIEnfermeira. Professora Permanente. Faculdade Pitágoras, Uberlândia, Minas Gerais, Brasil. E-mail: reginagava@yahoo.com.br. ORCID: https://orcid.org/0000-0001-5635-5757 Artigo proveniente da tese de doutorado "Matriz de Competências do enfermeiro que atua em Unidade de Centro Cirúrgico", apresentada em maio de 2019 à Universidade de São Paulo. 
Nessa direção, para atuação em UCC, o enfermeiro deve desenvolver tanto competências gerenciais como competências técnicas, assistenciais ${ }^{2}$. Sendo assim, repensar sobre as competências necessárias que possam balizar a sua prática profissional é aspecto relevante para refletir sobre lacunas de conhecimento, habilidades e atitudes destes trabalhadores.

Vale destacar que, em âmbito nacional, foram estabelecidas competências gerais para o enfermeiro ${ }^{3}$. No contexto internacional, na área de Centro Cirúrgico, destaca-se o sistema científico de avaliação de competências para enfermeiros cirúrgicos retratando desde competências relacionadas aos cuidados aos pacientes até aquelas específicas do gerenciamento ${ }^{4}$. Ainda, nessa direção, pesquisadores americanos atribuem as competências do enfermeiro de centro cirúrgico pautadas em conhecimento especializado, habilidades profissionais, atitudes e automotivação ${ }^{4,5}$.

Especificamente no contexto da gerência, o enfermeiro deve ser o mediador de situações conflituosas, realizando a negociação entre as diversas equipes no intuito de solucionar os problemas, pois situações de conflito são comuns em instituições de saúde onde trabalham profissionais com saberes diversificados. A falta de soluções para os conflitos pode trazer consequências desastrosas para a equipe e riscos para a saúde do usuário ${ }^{6}$.

Neste sentido, dentre as competências gerenciais do enfermeiro destaca-se aquela que pode ser capaz de gerenciar conflitos, estabelecendo um relacionamento harmonioso, estável, aqui denominada de competência relacional. O conceito de competência relacional tem suas origens na psicologia, sendo definida como a capacidade de formar e manter um relacionamento entre pares mais próximos, independentemente do tipo de relacionamento ${ }^{7}$. 0 conceito de competência relacional traduz a inteligência intelectual e emocional nas nossas relações conosco mesmo, com as pessoas, com o contexto e com a vida.

Nesse contexto, o enfermeiro gerente de unidades cirúrgicas precisa desenvolver a competência relacional para lidar com possíveis situações geradoras de desentendimento entre as equipes; isso implica em aprimorar capacidades como saber dialogar; e conhecer a dinâmica de trabalho para encontrar alternativas a fim de solucionar os problemas existentes, sejam eles relacionados a recursos humanos, materiais ou financeiros.

Acredita-se que a competência relacional, por estar diretamente relacionada à capacidade de trabalhar em equipe, parece ser uma das competências essenciais ao enfermeiro de UCC, e consiste na capacidade de se relacionar de forma eficaz com os outros. Assim, questiona-se: Como se estabelece a competência relacional entre os enfermeiros das UCC? Os enfermeiros utilizam esta competência para gerenciar conflitos no desenvolvimento de suas atividades?

Pensar na competência relacional como resultado de saberes cognitivos, habilidades e atitudes dos enfermeiros de UCC, deve auxiliar na superação das relações interpessoais tradicionais, no distanciamento entre os próprios trabalhadores de saúde, contribuindo consequentemente para o cuidado efetivo prestado aos usuários. Além disso, a não identificação desta competência profissional para setores específicos, como a UCC, pode acarretar problemas de relacionamentos e comportamentos na equipe, com consequência para o cuidado prestado.

Este artigo teve o objetivo de identificar e descrever como é desenvolvida a competência relacional no cotidiano do trabalho dos enfermeiros de UCC e identificar em que momento/situações o enfermeiro frequentemente utiliza essa competência.

\section{MÉTOdOS}

Estudo do tipo exploratório, utilizando-se da abordagem qualitativa. O cenário do estudo foi constituído por cinco instituições hospitalares privadas de um município do Estado de Minas Gerais. O período de coleta de dados ocorreu de janeiro a julho de 2018. Selecionaram-se enfermeiros de ambos os sexos atuando em UCC das instituições referidas com mais de seis meses de vínculo empregatício. Destaca-se que os enfermeiros possuíam carga horária semanal de 40 horas, relação de trabalho direta com equipe multiprofissional e a assistência propriamente dita. A população de enfermeiros era de 52 profissionais, porém participaram deste estudo 43 enfermeiros,, sendo excluídos enfermeiros em período de férias e afastados por motivo de doença.

Para a coleta de dados utilizou-se a técnica de Grupo Focal (GF), método que visa promover discussão em grupo entre os participantes acerca do tema ${ }^{8}$. Primeiramente realizou-se o período de sensibilização dos participantes, onde estes foram convidados, em abordagem pessoal por carta convite e via eletrônica (e-mail). Após, para compor o GF foram selecionados 6 a 10 enfermeiros sendo: um grupo de 8 enfermeiros no hospital I; dois grupos de 6 enfermeiros cada no hospital II; um grupo de 7 enfermeiros no hospital III; um grupo de 9 enfermeiros no hospital IV e um grupo de 7 enfermeiros no hospital V. Ademais, para composição dos grupos também se considerou o compartilhamento do mesmo local de trabalho, tendo em vista que favorece os relatos de experiências e necessidades permitindo interação na temática em questão. 
Salienta-se que os grupos foram realizados nas próprias instituições dos participantes, nas suas respectivas salas de reuniões ou auditório da empresa permitindo, assim, acesso facilitado aos profissionais por estarem no próprio local de trabalho.

No início da discussão foi aplicado um questionário aos participantes, contendo dados sociodemográficos, de formação e atuação profissional, tais como: sexo, idade, experiência, nível de tempo formação, pós-graduação, e tempo de atuação profissional na instituição e na Unidade Cirúrgica. Para o decorrer das discussões, utilizou-se duas questões norteadoras, elencadas anteriormente, bem como gravadores digitais para registro das informações.

Ressalta-se que os grupos focais foram conduzidos pelo pesquisador (moderador) e um auxiliar de pesquisa (observador). O moderador teve a função de manter o grupo em interação com segurança, passando confiança, sem criar conflitos, com a finalidade de obter dados acerca do tema da pesquisa e o observador teve a função de analisar a rede de interações presentes durante o processo grupal, visando descrever todas as situações identificadas pelos profissionais participantes ${ }^{8}$. Cada grupo teve a duração média de 50 a 55 minutos.

Após a coleta de dados, realizou-se a transcrição e a análise dos dados. Utilizou-se a análise temática indutiva, método que contribui para identificação, análise e possibilita uma descrição detalhada das informações, servindo como uma ferramenta útil e flexível de pesquisa ${ }^{9}$.

Realizou-se o estudo em conformidade com a resolução 466/2012, aprovado pelo Comitê de Ética em Pesquisa (CEP) da Instituição proponente sob o número CAAE 65960517.0.0000.5393, bem como, os participantes assinaram o Termo de Consentimento Livre e Esclarecido. Visando preservar o anonimato dos participantes optou-se por utilizar a letra "E" de enfermeiro, seguida do numeral arábico de acordo com a ordem cronológica crescente da realização dos grupos de cada hospital.

\section{RESULTADOS}

A amostra do estudo foi constituída por 43 enfermeiros que atuam nas UCC selecionadas. Observou-se a prevalência do sexo feminino ( $n=39 ; 90,6 \%)$ e da faixa etária entre 20 e 29 anos ( $n=17,2 ; 48,80 \%)$. Segundo o tempo de atuação na UCC houve prevalência de participantes atuando entre um e dois anos ( $n=8,4 ; 20,90 \%)$, sendo que 24 (58\%) afirmaram ter experiência profissional em unidades cirúrgicas de outros hospitais com média de tempo de três anos de atuação. Além disso, 30 (72\%) afirmaram terem sido preparados na graduação para atuar em Unidades Cirúrgicas, entretanto somente sete (16\%) afirmaram serem especialista para atuar especificamente nesta unidade e 10 (23,25\%) relataram terem especializações em outras áreas como UTI com ênfase em Urgência e Emergência, Auditoria, Gestão em Saúde e Controle de Infecção Hospitalar.

Os dados revelaram que a característica do relacionamento entre a equipe reflete na qualidade do cuidado prestado ao usuário. Nesse sentido, os participantes destacaram que a competência relacional se dá por meio de capacidades/aptidões a serem adquiridas pelos enfermeiros, destacando o gerenciamento de conflitos, a comunicação assertiva, gestão de pessoas e inteligência emocional.

\section{A competência relacional}

\section{Gerenciamento de conflitos}

Os participantes revelaram que para utilizar da competência relacional é vital que o profissional saiba gerenciar situações conflitantes dentro da equipe, sendo necessário o ajuste da carga horária dos trabalhadores, flexibilidade, saber manejar a situação e lidar com diferentes tipos de personalidades.

(...) quando a gente fala de competência relacional falamos de gerenciamento de conflito, porque são vários profissionais ao mesmo tempo, que a gente tem os fatores que não dependem só da gente. (E4 - Hospital III)

Gerenciar conflito é essencial para competência relacional... a forma de saber lidar com todas essas situações porque (...) nós trabalhamos com pessoas distintas, de personalidades distintas; então tem que saber a forma como vou lidar com X; com Y; como que eu vou conversar, até a forma de cobrar. Então isso tudo nós temos que ser ponderado e ter jogo de cintura. (E2 - Hospital III)

\section{Comunicação assertiva}

A comunicação também foi percebida como uma capacidade do profissional que deve estar agregada à competência relacional, pois, segundo os participantes, pode facilitar a resolução de questões conflitantes no trabalho, facilitando a resolução de situações de desarmonia entre as equipes.

(...) é acho que é a comunicação; é com uma comunicação assertiva que conseguimos lidar e amenizar diversos conflitos e assim ela tem tudo a ver com a competência relacional (E1 - Hospital II grupo focal I) 
Quando nós não conseguimos resolver com o médico aí nós conversamos com a diretora clínica para ela falar com eles de uma maneira mais imparcial, então é assim, se a nossa comunicação, nosso diálogo, não deu certo nós conversamos com ela e ai ela como diretora conversa e resolve com eles. (E4 - Hospital II grupo focal I)

Essa parte de comunicação que é a dificuldade grande, ter de lidar com alguns profissionais (...). Porque muitos trabalham do jeito deles, às vezes não sabem nos ouvir. (E3 - Hospital V)

Gestão de pessoas: dimensionamento de pessoal adequado

O enfermeiro de uma UCC deve ter competências para a gestão de pessoas, a exemplo, saber dimensionar o pessoal de enfermagem. O cálculo de dimensionamento de pessoal faz parte das suas funções e se mostra importante porque por meio dele justifica-se a necessidade de um quadro maior de trabalhadores para atendimento das demandas do setor. Os dados revelaram que ao utilizar-se do dimensionamento correto evita-se situações conflitantes, abrandando a precarização do trabalho e respeitando os intervalos e horas extras, bem como os direitos trabalhistas que se inadequados podem levar ao desgaste e estresse dos profissionais.

Seria uma reunião de gestão da escala...então de dizer a todos "olha vamos funcionar agora 12 horas e não mais 8 horas", pois o quantitativo de pessoal não dá para demanda de serviço, os funcionários dobram...e mesmo com boa comunicação sem o número adequado de funcionários poderia levar ao erro. (E4 - Hospital II grupo focal I)

Teve momentos que não houve enfermagem o suficiente no setor e eu cheguei ao médico e falei: as técnicas de enfermagem vão almoçar e o médico respondeu: como elas vão almoçar por quê?... então tive que pegar outros funcionários que tinham saído para o almoço e pedir para voltar antes do seu horário e outro que estava indo embora e eles tiveram que entrar em sala porque o serviço não pode parar, e isso levou a um conflito, pois a enfermagem já estava sobrecarregada e ainda tiveram que trabalhar mais, o que gerou discussão séria. (E1 - Hospital II grupo focal I)

\section{Inteligência emocional}

As enfermeiras apontaram que para o uso da competência relacional ser bem-sucedida é preciso ter inteligência emocional, ter controle de si, de suas emoções, para diante dos conflitos saber fazer uso da comunicação e se expressar de tal forma que seja compreendida.

E para fazer tudo isso (ter competência relacional) precisa ter calma e controle emocional por lidar com essas situações de cirurgia, cada dia uma diversidade diferente, o estresse do cirurgião, da equipe, imprevistos que podem acontecer no momento, então eu acho que é trabalhar sobre pressão e saber lidar com essas situações. (E5 - Hospital V)

O estresse emocional, a carga dos técnicos, e em conjunto com os médicos também, que nos colocam muita pressão por que tem que finalizar uma cirurgia por que já tem outra esperando, então esse controle tem que ser muito maior (...) é como se fossem metas que tem que cumprir. Junta também a parte de setor fechado, você fica 12 horas dentro de um setor, então a pessoa tem que ter controle. (E3 - Hospital V)

"Tem que ter controle emocional na hora dos conflitos, manter-se firme, com a equipe, com si mesma e com os outros setores, pois o tempo todo temos conflitos." (E6 - Hospital I)

Os enfermeiros revelaram ainda que para se ter inteligência emocional é preciso capacidade de se conhecer, conhecer a equipe e ser moderador, ser ponderado, ter bom senso para lidar com os diversos perfis profissionais que ali atuam.

Para eu ter competência relacional é preciso se conhecer primeiro para conseguir lidar com sua equipe, conhecer sua equipe e ser justo e ponderado, utilizando de proatividade, liderança e paciência. (E1- Hospital V)

O controle emocional é além de se autoconhecer é... (pausa) conhecimento da equipe também porque você tem que oferecer esse controle emocional. (E4 - Hospital IV)

\section{DISCUSSÃo}

Os dados evidenciaram população predominantemente feminina, consequência da cultura do trabalho da enfermagem, onde historicamente mulheres estão mais presentes nesta área. Corroborando a esses dados pesquisadores apontaram que nos primórdios as formas de assistência e cuidados ocorreram no ambiente doméstico, atividade que era privativa feminina, o que justificou a predominância desse gênero na profissão ${ }^{10}$.

O perfil profissional evidenciou participação em diversos cursos lato senso, mas a maioria não realizou cursos específicos em UCC. Os resultados mostram que a formação acadêmica especializada para atuação em UCC não foi considerada relevante na seleção dos profissionais, visto que somente pequena porção dos profissionais possuía esse conhecimento mais aprofundado.

Entretanto, faz-se relevante refletir a procura constante por cursos de pós-graduação pela enfermagem, fato este que pode ser atribuído pela insegurança e falta de habilidade advinda com a formação. Evidências apontam que o 
enfermeiro chega ao mercado com preparo limitado para enfrentar sua realidade de trabalho e, por isso, muitas vezes, busca por aprimoramento profissional após a formação acadêmica ${ }^{11}$. Ademais, outro estudo revelou que o tempo de trabalho e experiência em setor de alta complexidade semelhante ao tempo de atuação na própria instituição, demonstra que grande parte dos profissionais admitidos são contratados desde o início para atuar no setor de alta complexidade, sendo muitos deles jovens e recém-formados ${ }^{12}$, corroborando com os resultados em que se constatou enfermeiros jovens, justificado pela característica dos tempos atuais, em que estes, cada vez mais cedo adentram em universidades.

Sabe-se que na UCC a capacidade para trabalhar em equipe é essencial ao enfermeiro, considerando sua constante interação com uma equipe multiprofissional. Tal interação pode estar presente e ser adequada quando ocorrer o desenvolvimento da competência relacional. Nessa direção, este estudo evidenciou que a competência relacional pode acontecer por meio da capacidade de gerenciar conflitos, comunicação assertiva, dimensionamento de pessoal adequado e inteligência emocional.

Os conflitos permeiam o ambiente laboral podendo acarretar em desgaste e prejuízos na assistência. Desta forma, para gerenciar situações conflitantes é vital o ajuste da carga horária dos trabalhadores, saber manejar as situações e lidar com diferentes personalidades. Pesquisas revelam que o conflito no trabalho foi encontrado como fator de estresse dominante e que pode levar ao Burnout ${ }^{13}$.

Indo de encontro a competência relacional, sabe-se que para gerenciar conflitos é imprescindível o uso da comunicação assertiva. A este respeito, evidências apontam que a liderança eficaz estimula a comunicação entre os membros da equipe, e com o aumento da comunicação, os membros da equipe podem adquirir informações relevantes sobre os colegas, como suas competências pessoais. Isso, por sua vez, leva os membros a formar julgamentos sobre a confiabilidade dos colegas. Assim, a informação contida na comunicação entre os membros da equipe formaria a base para a confiança se desenvolver nessas novas equipes ${ }^{14}$. Nesse sentido, construir uma comunicação eficaz entre os membros da equipe se mostra necessário para a colaboração entre os profissionais.

Sabe-se que o simples ato de comunicar-se não é uma tarefa tão simples, uma vez que normalmente os indivíduos estão fechados dentro de si mesmo, pela educação recebida, relacionamentos conflitantes, timidez e fobia social, e esses fatores podem interferir na forma de se comunicar e interagir em grupos sociais distintos. Estudos ${ }^{15}$ evidenciaram $^{-1}$ que cursos sobre habilidades de comunicação deveriam ser obrigatórios durante o treinamento de pessoal devendo ser incorporados em todos os níveis de educação permanente, desde iniciantes a enfermeiros com experiência profissional.

Sobretudo, para que a assistência cirúrgica ocorra de maneira eficaz, é essencial uma gestão de pessoas eficiente por parte do enfermeiro, preocupando com o dimensionamento adequado de sua equipe, pois o número reduzido de trabalhadores contribui para maior sobrecarga e estresse, gerando conflitos, que podem interferir na qualidade do cuidado e, consequentemente, afetando a competência relacional que deve estar presente em uma UCC ${ }^{16}$.

A este respeito, os enfermeiros revelaram que o quadro reduzido da equipe pode ainda ocasionar erros adversos, mesmo que a comunicação se faça presente em todo o processo. Assim, com o dimensionamento adequado evita-se a sobrecarga de trabalho, que apresenta riscos de estresse aos profissionais de UCC. De acordo com pesquisadores o ambiente cirúrgico é considerado estressante devido a diversos fatores de trabalho e condições físicas indesejáveis, como ruído, luz, calor, umidade, alta carga de trabalho e baixo número de funcionários ${ }^{17}$.

Portanto, o quadro suficiente de profissionais de enfermagem se faz imprescindível para assim desenvolver a competência relacional. O dimensionamento deve ser calculado pelo enfermeiro respeitando o tempo gasto para cada cirurgia e para higienização das salas operatórias, a classificação das cirurgias e o número de horas para prestação de cuidados para cada paciente, sendo que em 24 horas é necessário um enfermeiro para cada três salas operatórias, um técnico de enfermagem para cada sala operatória assumindo a circulação da mesma, e um técnico de enfermagem para realizar instrumentação de cada sala operatória ${ }^{18}$

Pesquisadores $^{19}$ reforçam que os cálculos de dimensionamento são fundamentais para o planejamento e organização dos cuidados; além disso, o uso de recursos e programas informatizados contribui para facilitar a realização desta atividade.

Ainda, este estudo revelou a importância da inteligência emocional para garantia da competência relacional. O uso do controle emocional é relevante no processo de trabalho da equipe cirúrgica, pois se trata de uma área complexa com a presença de situações estressantes aos profissionais que ali atuam. A inteligência emocional oferece uma estrutura para se aprimorar a colaboração, comportamentos positivos durante o conflito e relacionamentos saudáveis no ambiente de atendimento clínico. É um importante fator que pode medir o desempenho dos indivíduos em suas vidas profissionais, aumentar ou diminuir seu sucesso, contribuir para a mensuração das qualidades gerenciais, e melhorar a comunicação e interação organizacional ${ }^{16}$, se mostrando como componente necessário para construir a competência relacional. 
Nesse sentido, para o aprimoramento dessa habilidade, é necessário elaborar estratégias que consolide a inteligência emocional. Estudos indicam que criação de situações apropriadas para os enfermeiros expressarem suas emoções negativas pode reduzir sua tensão emocional e impedir a exaustão e a frustração ${ }^{17}$. Sendo assim, há necessidade de programas de treinamento projetados para melhorar o gerenciamento de conflitos e a inteligência emocional dos enfermeiros, a fim de gerenciar efetivamente os conflitos entre eles, muitas vezes inevitável nas instituições de saúde ${ }^{16}$.

Portanto, a competência relacional pode ser trabalhada e aprimorada por meio dessas habilidades e por meio da educação permanente, contribuindo para elevar o desempenho da equipe, que consequentemente reflete em melhora da qualidade do cuidado prestado ao usuário.

Este estudo apresenta a limitação de ter sido realizado somente com enfermeiros, e em unidades cirúrgicas de instituições privadas, não podendo generalizar os resultados encontrados para outras realidades. Assim, sugere-se que sejam realizados estudos em instituições públicas, bem como com outros membros da equipe de saúde vinculados a Unidades Cirúrgicas.

\section{CONSIDERAÇÕES FINAIS}

O estudo permitiu evidenciar a competência relacional como imprescindível para realização do trabalho em UCC. Estas unidades apresentam características que trazem riscos de desgaste físico e emocional aos profissionais, o que faz com que situações conflitantes apareçam rotineiramente. Nesse sentido, para lidar de forma assertiva com essas adversidades, aprimorar a competência relacional se mostrou substancial utilizando para tal a capacidade de gerenciar conflitos, comunicação assertiva, gerenciar recursos humanos e apresentar inteligência emocional.

Embora os indivíduos sejam seres sociáveis, todo relacionamento é complexo, pois as pessoas têm diferentes personalidades e comportamentos. E em um contexto competitivo, como o ambiente de trabalho hospitalar, esses fatores são ainda mais agravantes, pois refletem diretamente nos resultados e performance do profissional com consequências para a instituição.

No ambiente cirúrgico, onde existem atividades com alto nível de complexidade, além de equipe multiprofissional torna-se evidente a necessidade de gestores e profissionais refletirem sobre o seu papel e as competências necessárias para a interprofissionalidade, como desenvolver capacidades para a competência relacional, a fim de alcançar a eficiência na sua atuação. Este estudo deve permitir a reflexão dos centros formadores, para que reconheçam a importância de adaptar as estruturas pedagógicas para atender as demandas e exigências do atual mercado de trabalho, bem como deve provocar mudanças na prática clínica, visto que a construção e manutenção da competência relacional proporciona uma melhora na qualidade do cuidado prestado.

\section{REFERÊNCIAS}

1. Rocha DR, Ivo OP. Nursing care in the preoperative period and its influence on postoperative. Rev. Enferm. Contemp. [Internet]. 2015 [cited 2020 Jul 15]; 4(2):170-8. DOI: http://dx.doi.org/10.17267/2317-3378rec.v4i2.631

2. 2.Zhao F, Li L, Li J,Wang C. The Role of Core Competence Training in the Training of Junior Nurses in Operating Room. In: $3^{\text {rd }}$ International Conference on Economics, Management Engineering and Education Technology [Internet]; 2019 may 18-19; Suzhou, China. United Kingdom: Francis Academic Press; 2019 [cited 2020 Jul 15]. p.1443-6. Available from: https://webofproceedings.org/proceedings_series/article/artld/7906.html\#location

3. Leal LA, Soares MI, Silva BR, Brito LJS, Bernardes A,Henriques SH. Professional competencies for hospital nurses: a documentar analysis. Rev. enferm. Cent.-Oeste Min. [Internet]. 2019 [cited 2020 Jul 15]; 9:e3249. DOI: https://doi.org/10.19175/recom.v9i0.3249

4. Wang Y, Xiong L, Ma Y, Gao X, Fu W. Construction of competency evaluation measures for operating room nurses. Chin. Nurs. Res. [Internet]. 2016 [cited 2020 Jul 15]; 3(4):181-4. DOI: https://doi.org/10.1016/j.cnre.2016.07.001

5. Santos APA, Camelo SHH, Santos FC, Leal LA, Silva BR. Nurses in post-operative heart surgery: professional competencies and organization strategies. Rev. esc. enferm. USP [Internet]. 2016 [cited 2020 Jul 15]; 50(3):474-81. DOI: https://doi.org/10.1590/S0080-623420160000400014

6. Camelo SHH, Soares MI, Chaves LDP, Rocha FLR, Silva VLS. Nurse managers at a teaching hospital: training, responsibilites and challenges. Rev. enferm. UERJ. [Internet]. 2016 [cited 2020 Jul 15]; 24(3):e11637. DOI: https://doi.org/10.12957/reuerj.2016.11637

7. Cashen KK, GrotevantHD. Relational competence in emerging adult adopters: conceptualizing competence in close relationships. J. Adult Dev. [Internet]. 2019 [cited 2020 Jul 15]; 27:83-94. DOI: https://doi.org/10.1007/s10804-019-09328-x

8. Nyumba TO, Wilson K, Derrick CJ, Mukherjee N. The use of focus group discussion methodology: Insights from two decades of application in conservation. Methods Ecol. Evol. [Internet]. 2018 [cited 2020 Jul 15]; 9:20-32. DOI: https://doi.org/10.1111/2041-210X.12860

9. Braun V,Clarke V. Using thematic analysis in psychology. Qual. Res. Psychol [Internet]. 2006 [cited 2020 Jul 15]; 3(2):77-101. DOI: https://doi.org/10.1191/1478088706qp063oa 
10. Galbany-EstragueP, Comas-D'argemir D. Care, Autonomy, and Gender in Nursing Practice: A Historical Study of Nurses' Experiences. J. Nurs. Res. [Internet]. 2017 [cited 2020 Jul 15]; 25(5):361-67. DOI: https://doi.org/10.1097/JNR.0000000000000184

11. Leal LA, Soares MI, Silva BR, Bernardes A, Camelo SHH. Clinical and management skills for hospital nurses: perspective of nursing university students. Rev. bras. Enferm. [Internet]. 2018 [cited 2020 Jul 15]; 71(Suppl4):1514-21. DOI: https://doi.org/10.1590/0034-7167-2017-0452

12. Santos JLG, Erdmann AL. Governance of professional nursing practice in a hospital setting: a mixed methods study. Rev. LatinoAm. Enferm. [Internet]. 2015 [cited 2020 Jul 15]; 23(6):1024-32. DOI: https://doi.org/10.1590/0104-1169.0482.2645

13. 13.Salamat ASA, Yusof NM, Ibrahim S, Zamhury N, Ramli SI. Job Stressors and Job Burnout. In: The $6^{\text {th }}$ International Conference on Public Policy and Social Science (ICOPS) [Internet]. 2017 marc 8-9; Cawangan, Kedah, Malaysia. Malaysia: ICOPS; 2017 [cited 2020 Jul 15]. p.1087-95. Available from: https://www.researchgate.net/publication/322076604_FULL_PAPER_ICOPSS2017

14. Boies K, Fiset J, Gill H. Communication and trust are key: unlocking the relationship between leadership and team performance and creativity. Leadersh. Q. [Internet]. 2015 [cited 2020 Jul 15]; 26(6):1080-94. DOI: https://doi.org/10.1016/j.leaqua.2015.07.007

15. Song $\mathrm{H}$, Choi J, Son $\mathrm{Y}$. The relationship between professional communication competences and nursing performance of critical care nurses in South Korea. Int. J. Nurs. Pract. [Internet]. 2016 [cited 2020 Jul 15]; 23(5):e12576. DOI: https://doi.org/10.1111/ijn.12576

16. Basogul C, Ozgur G. Role of emotional intelligence in conflict management strategies of nurses. Asian Nurs. Res. [Internet]. 2016 [cited 2020 Jul 15]; 10(10):228-33. DOI: https://doi.org/10.1016/j.anr.2016.07.002

17. Soltanmoradi $Y$, Ansari A, Heidari Sh. Occupational stress among operating room nurses of hospitals affiliated to Kerman universities of medical sciences, Iran (2016): A cross-sectional study. JOHE [Internet]. 2016 [cited 2020 Jul 15]; 6(4): 225-33. DOI: https://doi.org/10.29252/johe.6.4.225

18. Conselho Federal De Enfermagem (Cofen). Resolução $n^{\circ}$. 543, de 18 de abril de 2017. Atualiza e estabelece parâmetros para o dimensionamento do quadro de profissionais de enfermagem nos serviços/locais em que são realizadas atividades de enfermagem. Brasília (DF); 2017 [cited 2020 Jul 15]. Available from: http://www.cofen.gov.br/resolucao-cofen5432017_51440.html

19. Vandresen L, Pires DEP, Lorenzetti J, Andrade SR. Classification of patients and nursing staff's sizing: contributions of a management technology. Rev. Gaúcha Enferm. [Internet]. 2018 [cited 2020 Jul 15]; 39:e2017-0107. DOI: https://doi.org/10.1590/1983-1447.2018.2017-0107 